\title{
Rapid Method of Obtaining Area Under Curve for Any Compartment of Any Linear Pharmacokinetic Model in Terms of Rate Constants ${ }^{1}$
}

\author{
John G. Wagner ${ }^{2,3}$
}

Received Jan. 28, 1976

\section{INTRODUCTION}

The classical method of obtaining areas in terms of kinetic constants involves the following steps: (a) writing the differential equations for the model; (b) obtaining the Laplace transform $\left(a_{i}\right)$ for the amount in a given compartment at time $t\left(A_{i}\right)$; (c) taking the antitransform which provides the expression for $A_{i}$ which is a polyexponential equation; (d) integrating the polyexponential equation between the limits of $t=0$ and $t=\infty$; and (e) simplifying the result. The last step in this sequence often involves horrendous algebra.

\section{THEORETICAL}

The Laplace transform of a function, $F(t)$, is obtained as indicated by

Supported in part by Public Health Service Grant 5-P11-GM 15559.

${ }^{1}$ Editorial Note: Dr. Wagner's article is published as a Scientific Commentary for the sake of the reader uninformed in basic properties of the LaPlace transform. It represents a reiteration of a basic theorem of LaPlace transforms. The final value theorem can be found in $\mathbf{P}$. A. McCollum and B. F. Brown, LaPlace Transform Tables and Theorems, Holt, Rinehart and Winston, New York, pp. 73-74, 1965. It may also be found in H. S. Carslow and J. C. Jaeger, Operational Methods in Applied Mathematics, Dover Publications, New York, pp. 255-256, 1963.

${ }^{2}$ College of Pharmacy and Upjohn Center for Clinical Pharmacology, The University of Michig:n, Ann Arbor, Michigan 48109.

${ }^{3}$ Address reprint 1 equests to Dr. John G. Wagner, Upjohn Center for Clinical Pharmacology, The University oi Michigan Medical Center, Ann Arbor, Michigan 48109. 


$$
L[F(t)]=a_{i}=\int_{0}^{\infty} F(t) e^{-s t} d t
$$

When $s=0$, then $e^{-s t}=1$, and

$$
\left(a_{i}\right)_{s=0}=\int_{0}^{\infty} F(t) d t
$$

In linear pharmacokinetics, $F(t)$ is given by

$$
F(t)=V_{p} \int_{0}^{\infty} C_{i} e^{-\lambda_{i} t}=\int_{0}^{\infty} A_{i} e^{-\lambda_{i} t}
$$

for the plasma or reference compartment, where $V_{p}$ is the volume of that compartment, the $C_{i}$ 's and $A_{i}$ 's are coefficients with dimensions of concentration and mass, respectively, and the $\lambda_{i}$ 's are either eigenvalues or microscopic rate constants of the particular model.

Dost's "law of corresponding areas" (1) may be stated as follows: the ratio of the area beneath the blood level-time curve after oral administration to that following intravenous administration of the same dose is a measure of the absorption of the drug administered. This may be expressed mathematically as

$$
F=\int_{0}^{\infty} C_{p}^{\text {p.o. }} d t / \int_{0}^{\infty} C_{p}^{\mathrm{i} . v .} d t
$$

In equation $4, F$ symbolizes the fraction of the dose which is absorbed (hence is the bioavailability factor due to incomplete absorption), $C_{p}^{\text {p.o. }}$ is the plasma concentration at time $t$ after oral administration, and $C_{p}^{i . v}$ is the plasma concentration at time $t$ after intravenous administration.

Now, Dost's law should be replaced by

$$
F F^{*}=D_{\text {i.v. }} \int_{0}^{\infty} C_{p}^{\text {p.o. }} d t / D_{\text {p.o. }} \int_{0}^{\infty} C_{p}^{\text {i.v. }} d t
$$

In equation $5, D_{\text {i.v. }}$ represents the dose given intravenously, $D_{\text {p.o. }}$ represents the dose given orally and $F^{*}$ is the bioavailability factor due to the so-called first-pass effect. When dealing with linear pharmacokinetic models, the value of $F^{*}$ is obtained by assuming $F=1$ and $D_{\text {i.v. }}=D_{\text {p.o. }}$ and then substituting the appropriate values for the two areas into equation 5 and simplifying, if necessary. 


\section{EXPERIMENTAL}

Figure 1 shows the schematic diagrams of 6 linear pharmacokinetic models. Table I lists the Laplace transforms for the amounts in the designated plasma compartment (signified by $V_{p}$ being written below that compartment) after both oral, $a_{p}^{\text {p.o. }}$, and intravenous, $a_{p}^{\text {i.v. }}$, administration, the corresponding areas, and the value of $F^{*}$ for the model. The areas obtained by the application of equation 2 were all checked by the classical method of integrating the polyexponential equation for the amount in the plasma compartment as a function of time and agreement was obtained in each case.

Since the products of the $\lambda_{i}$ 's appearing in the area expressions cancel when the ratio of the oral to the intravenous area is made to obtain $F^{*}$, it is not necessary to know what the $\lambda_{i}$ 's mean in terms of the microscopic rate

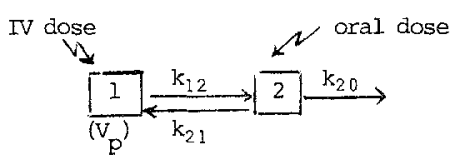

Model I

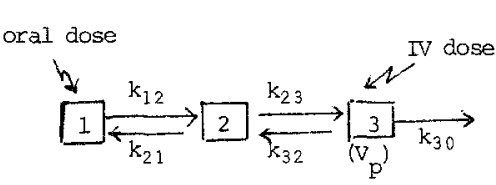

Model III

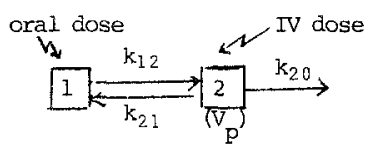

Model II

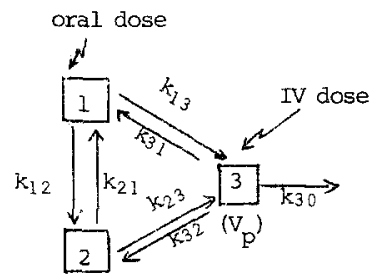

Model IV

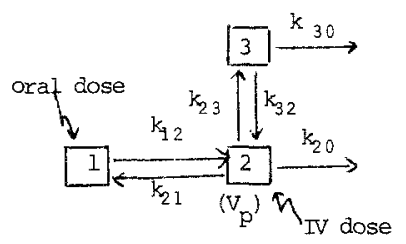

Model V

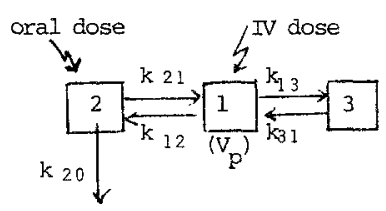

Model VI

Fig. 1. Schematic diagrams of six linear pharmacokinetic models. 


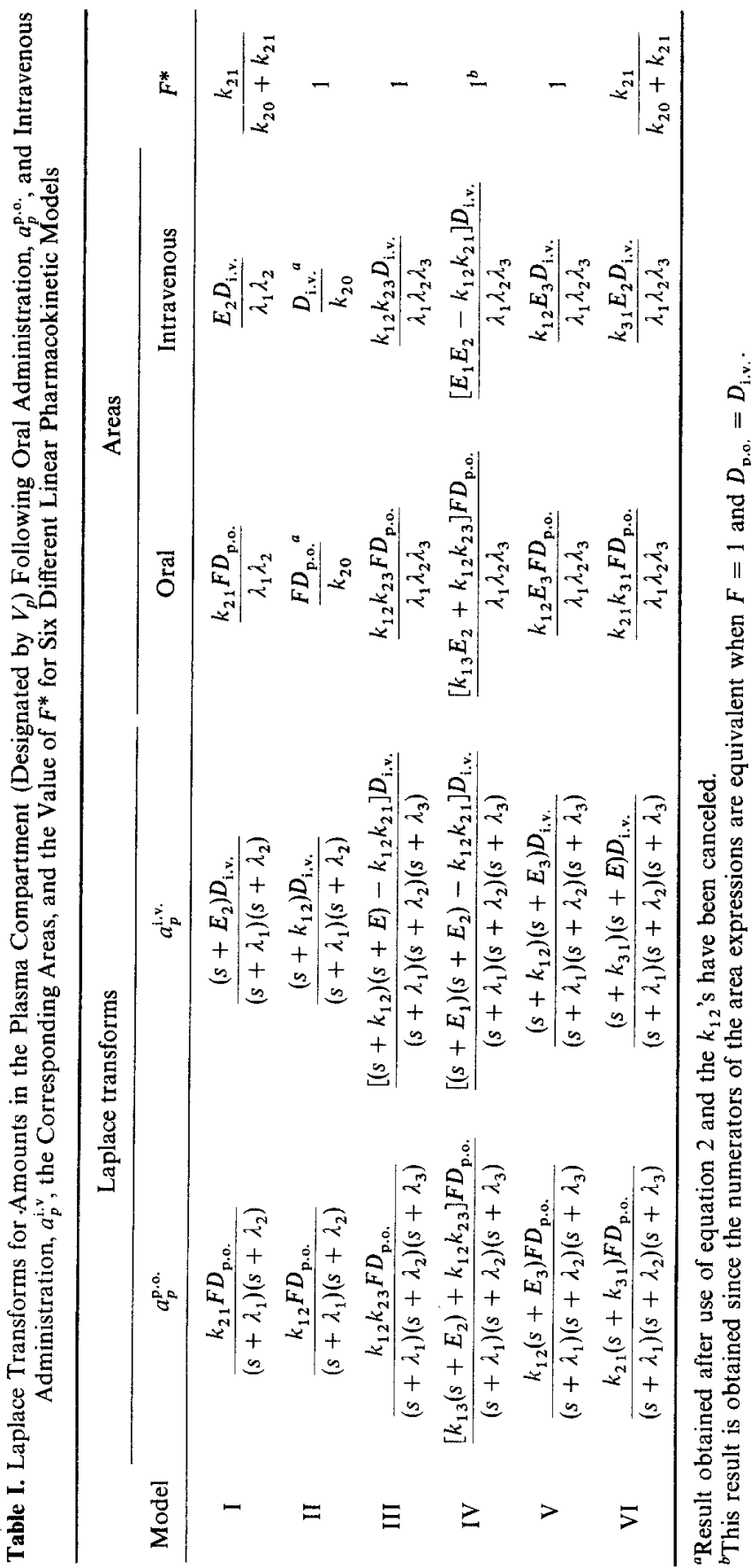


constants to obtain the value of $F^{*}$ for any particular model. However, for the models shown in Fig. 1, the products are as follows:

$$
\begin{gathered}
\text { Models I and II: } \lambda_{1} \lambda_{2}=k_{12} k_{20} \\
\text { Model III: } \quad \lambda_{1} \lambda_{2} \lambda_{3}=k_{12} E_{2} E_{3}-k_{12} k_{23} k_{32}-k_{12} k_{21} E_{3}
\end{gathered}
$$

where $E_{2}=k_{21}+k_{23}$ and $E_{3}=k_{32}+k_{30}$.

$$
\text { Model IV: } \begin{aligned}
\lambda_{1} \lambda_{2} \lambda_{3}= & E_{1} E_{2} E_{3}-k_{23} k_{32} E_{1}-k_{12} k_{21} E_{3}-k_{13} k_{21} k_{32} \\
& -k_{12} k_{23} k_{31}-k_{13} k_{31} E_{2}
\end{aligned}
$$

where $E_{1}=k_{12}+k_{13}, E_{2}=k_{21}+k_{23}$, and $E_{3}=k_{30}+k_{31}+k_{32}$.

$$
\text { Model V: } \lambda_{1} \lambda_{2} \lambda_{3}=k_{12} E_{2} E_{3}-k_{12} k_{23} k_{32}-k_{12} k_{21} E_{3}
$$

where $E_{2}=k_{20}+k_{23}+k_{21}$ and $E_{3}=k_{30}+k_{32}$.

$$
\text { Model VI: } \quad \lambda_{1} \lambda_{2} \lambda_{3}=E_{1} E_{3} k_{31}-k_{12} k_{21} k_{31}-k_{13} k_{31} E_{2}
$$

where $E_{1}=k_{12}+k_{13}$ and $E_{2}=k_{20}+k_{21}$.

\section{DISCUSSION}

The above method is clearer and more in keeping with acceptable pharmacokinetic theory than the method proposed by Nüesch (2) to make the correction (i.e., find $F^{*}$ ) that makes Dost's law valid for a given compartment model.

However, in the real world (as contrasted to the abstract world of models) the only way to prove that Dost's law is applicable to a particular drug is to show that, with some type of dosage form, $F F^{*}=1$ when one measures the drug in plasma after both oral and intravenous administration and applies equation 5. Such a result implies that the oral dose was completely absorbed (i.e., $F=1$ ) and that for conditions existing in the body $F^{*}=1$. If the oral area is less than the intravenous area, one really cannot determine whether this was caused by $F<1$ or $F^{*}<1$ or both being less than unity. This is because the bioavailability factors are confounded (i.e., appear as a product, $F F^{*}$, in equation 5). It has been recognized for some time that the value of $F^{*}$ can be so close to unity (e.g., when $k_{21} \gg k_{20}$ in models I and VI of Fig. 1) that, with the errors involved in plasma assays and in estimating the areas, one cannot distinguish the value from unity.

\section{REFERENCES}

1. F. H. Dost. Grundlagen der Pharmacokinetik, 2nd ed., Thieme, Stuttgart, 1968.

2. E. Nüesch. Proof of the general validity of Dost's law of corresponding areas. Eur. J. Clin. Pharmacol. 6:33-43 (1973). 